\title{
Can we talk? The art (and science) of handoff conversation
}

\author{
Julie K Johnson, ${ }^{1}$ Vineet M Arora ${ }^{2}$
}

\begin{abstract}
${ }^{1}$ Department of Surgery, Center for Healthcare Studies, Institute for Public Health and Medicine, Feinberg School of Medicine, Northwestern University, Chicago, Illinois, USA ${ }^{2}$ Department of Medicine, University of Chicago, Chicago, Illinois, USA
\end{abstract}

\section{Correspondence to}

Dr Julie K Johnson, Department of Surgery, Center for Healthcare Studies, Institute for Public Health and Medicine, Feinberg School of Medicine, Northwestern University, 633 N. St. Clair, 20th Floor, Chicago, IL 60611, USA; julie.k.johnson@ northwestern.edu

$\mathrm{JKJ}$ is an Associate Editor at BMJ Qual Saf.

Accepted 4 August 2015

\section{SLinked}

http://dx.doi.org/10.1136/ bmjqs-2014-003694

- http://dx.doi.org/10.1136/ bmjqs-2014-003853

- http://dx.doi.org/10.1136/ bmjqs-2015-004181

\section{CrossMark}

To cite: Johnson JK, Arora VM. BMJ Qual Saf 2016;25:63-65.
Art and science have their meeting point in method.

-Earl Edward George Bulwer-Lytton, Caxtoniana (1875), 303

The handoff or handover of patient care is not just a simple act of communication. It is a complex exchange of patient information that increases the likelihood of safe and effective care. We know that these transfers of care are a vulnerable link in patient care that is associated with preventable adverse events. It is especially concerning that effective handoff communication is not regularly or systematically taught to health professionals.

It is the interaction between the 'sender' and the 'receiver' that matters. Accurate transmission from the sender is not sufficient and without questions may result in a handoff 'telegram'. ${ }^{1}$ The solution to the telegram problem lies partly with the sender and the information they transmit and with the receiver and their ability to ask the right questions-the handoff conversation, so to speak.

Three papers in this issue of $B M J$ Quality and Safety encourage us to think about the art, as well as the science, of the handoff conversation. ${ }^{2-4}$ In a study conducted at one Veteran's Affairs (VA) Medical Center in the USA, Bergman and colleagues ask whether our efforts to improve have created an over-reliance on technology and standardisation-that is, structured handoff frameworks or checklists focus on the mechanical aspects, but don't take into account the patientspecific context. ${ }^{3}$ They argue that in actuality we have created another vulnerability and that we may miss contextually sensitive information about anticipated events. "Mr Smith's been our problem child": anticipatory Management Communication (AMC) in VA end-of-shift medicine and nursing handoffs' defines AMC as "a family of conversational strategies in which one party (typically the outgoing healthcare professional) informs or alerts another party (typically the responsible incoming healthcare professional) about the current status and projected course of events that are likely to be encountered in assuming the care of the patient".

Ultimately, their results come as sobering news, which still make us ask about how best to structure handoff conversations and how best to train the senders and receivers, and how to assess the quality of handoffs. Bergman and colleagues found that 'Heads-up' information was the most frequent type of AMC across all nursing and resident handoff dyads. Indirect instructions-"he has a big old rash on his bottom..."-occurring in all the nursing handoffs and more than half the resident handoffs are vague and leave opportunity for miscommunication and potential harm. The concept of mental models-the "lens in which we see reality" 5 - comes into play here. Do the sender and the receiver in the handoff dyad have similar or different mental models? Although systems thinking teaches us that shared mental models are necessary for improvement work, in the handoff conversation, different mental models may lead to more discussion and a co-construction of clinical understanding. ${ }^{1}$

Indirect instructions are an example of how language in the handoff is used formally and informally. The assumption that AMC was given does not mean the handoff conversation was well executed. In addition to AMC from senders, questions from receivers play an important role in co-constructing the course, direction and outcome of the handoff. As part of the same study, but in a separate paper included in this issue, "Anybody on this list that you're more worried about?" Qualitative analysis exploring the functions of questions during end of shift handoffs', O'Brien and colleagues conducted a qualitative analysis of the 
function of questions during end-of-shift handoffs. ${ }^{2}$ Their analysis revealed that, not surprisingly, the vast majority of questions are asked by the incoming health professional to help elicit more information to understand the individual patient and to plan care accordingly.

While neither of these papers report the training provided to conduct effective handoff conversations, the findings suggest that training could be improved. Moreover, while these studies highlight that handoff of patient care is best envisioned as a conversation, the quality of the handoff depends on the content of that conversation. Better content sets the stage for a better conversation. Given that most handoffs occur as a one-way passive dump of information without active listening behaviours, we need a new paradigm that teaches clinicians how to 'converse' during handoffs.

A third paper in this issue provides an example of what such a new paradigm might look like. In 'Advancing the next generation of handover research and practice with cognitive load theory', Young et al ${ }^{4}$ argue that practice innovations, to date, have been 'first-generation strategies' focused on structured protocols, mnemonics, and so on. While these strategies have led to significant improvements in education and clinical outcomes, further improvement, what they call 'second-generation strategies', will require a deeper understanding of human cognitive abilities, particularly the factors that affect working memory, the key memory subsystem related to handover.

Cognitive learning theory highlights three factors that consume limited working memory resources: intrinsic load, the work of the task itself, which is affected by, for instance, the complexity of patients; extraneous load, such as interruptions or distractions, which are task unrelated; and germane load, the work of processing the information, reflected, for example, in the need to ask clarifying questions. Young encourages the use of cognitive learning theory to consider not just the questions that O'Brien analysed (the germane load), but how the sum of the three types of load compares to the clinician's working memory capacity. Performance suffers when the individual has limited working memory resources to allocate to germane load. Young recommends that we titrate the intrinsic load by reducing complexity (eg, with a checklist), reduce the extraneous load by minimising distractions and optimise the germane load to allow co-construction of the sender's and receiver's mental model of the patient.

Together, these papers remind us that while adopting standardise tools and checklists may come relatively easily, it remains unclear how well these tools will promote more effective handoff conversations. One promising development is the introduction of the I-PASS (illness, severity, patient summary, action list, situation awareness and contingency plans, and synthesis by receiver) bundle, which has been associated with a reduction in preventable adverse events. ${ }^{6}$ While these results are encouraging, the bundled intervention included both handoff standardisation and team training, leaving us to wonder which ingredient(s) improved outcomes. Moreover, not all sites benefited from the implementation of I-PASS, highlighting the critical importance of organisational culture and context.

In addition to interventions to standardise handoffs, the current prevailing paradigm of handoff education 'invests in the sender', ignoring the critical role that receivers must play. Research from our group has demonstrated that even in ideal conditions (limited interruptions, supervision, education) the most important piece of information was not relayed to the receiver $60 \%$ of the time despite senders believing they had conveyed this information. ${ }^{7}$ Among hospitalist handoffs, we find that receivers are not activated, often assuming a passive role such as 'back channelling' with head nods or saying 'uh huh', which give the illusion of understanding. Yet, our geriatricians caution us that even patients with dementia are capable of back channelling. Moreover, all hospitalist handoffs were plagued with interruptions-and while pagers were prevalent, side conversations that resulted from tangential questions derailed handoffs. ${ }^{8}$

As we consider the future of research to advance our understanding and improvement of handoffs, it is important to consider both the 'art' and the 'science' of the handoff conversation. How do we think about an effective handoff conversation, not just for one patient, but for a group of patients of differing levels of illness, acuity and urgency? Our sickest patients and those at greatest risk will require a different level of handoff. Further work is needed to understand how senders can guard against the 'egocentric heuristic', where they believe that the receiver has the same information because a handoff was performed. What are the best ways to empower receivers to be resilient against threats to their understanding such as excessive workload, interruptions or their own fatigue and burnout? What characteristics of senders and receivers may impact their ability to have an effective conversation? What prior training or experiences influence clinician ability to converse effectively? And lastly, what is the return on investment of training clinicians to have better handoff conversations not just in terms of traditional safety outcomes, but in terms of efficiency, value and patient experience.

Through reframing handoffs as a conversation, we certainly still have much to talk about.

Twitter Follow Julie Johnson at @JulieJKJohnson and Vineet Arora at@futuredocs

Provenance and peer review Not commissioned; internally peer reviewed. 


\section{REFERENCES}

1 Cohen MD, Hilligoss B, Kajdacsy-Balla Amaral AC. A handoff is not a telegram: an understanding of the patient is co-constructed. Crit Care 2012;16:303.

2 O'Brien CM, Flanagan ME, Bergman AA, et al. "Anybody on this list that you're more worried about?" Qualitative analysis exploring the functions of questions during end of shift handoffs. BMJ Qual Saf 2016;25:76-83.

3 Bergman AA, Flanagan ME, Ebright PR, et al. "Mr Smith's been our problem child today...": anticipatory management communication (AMC) in VA end-of-shift medicine and nursing handoffs. BMJ Qual Saf 2016;25: 84-91.
4 Young JQ, Wachter RM, ten Cate O, et al. Advancing the next generation of handover research and practice with cognitive load theory. BMJ Qual Saf 2016;25:66-70.

5 Senge P. The fifth discipline. New York: Doubleday, 1990.

6 Starmer AJ, Spector ND, Srivastava R, et al. Changes in medical errors after implementation of a handoff program. N Engl J Med 2014;371:1803-12.

7 Chang VY, Arora VM, Lev-Ari S, et al. Interns overestimate the effectiveness of their hand-off communication. Pediatrics 2010;125:491-6.

8 Greenstein EA, Arora VM, Staisiunas PG, et al. Characterising physician listening behaviour during hospitalist handoffs using the HEAR checklist. BMJ Qual Saf 2013;22:203-9. 